日本原子力研究開発機構機関リポジトリ

Japan Atomic Energy Agency Institutional Repository

\begin{tabular}{|c|l|}
\hline Title & $\begin{array}{l}\text { First-principles study of solvent-solute mixed dumbbells in } \\
\text { body-centered-cubic tungsten crystals }\end{array}$ \\
\hline Author(s) & Suzudo Tomoaki, Tsuru Tomohito, Hasegawa Akira \\
\hline Citation & Journal of Nuclear Materials,505,p.15-21 \\
\hline Text Version & Preprint \\
\hline URL & https://jopss.jaea.go.jp/search/servlet/search?5061097 \\
\hline DOI & https://doi.org/10.1016/j.jnucmat.2018.03.052 \\
\hline Right & Elsevier \\
\hline
\end{tabular}




\title{
First-principles study of solvent-solute mixed dumbbells in body-centered-cubic tungsten crystals
}

\author{
Tomoaki Suzudo $^{*, a}$, Tomohito Tsuru ${ }^{\mathrm{b}, \mathrm{c}}$, Akira Hasegawa ${ }^{\mathrm{d}}$ \\ ${ }^{a}$ Center for Computational Science and e-Systems, Japan Atomic Energy Agency 2-4 Shirane Shirakata \\ Tokai-mura, 319-1195, Japan \\ ${ }^{b}$ Center for Basic Nuclear Research, Japan Atomic Energy Agency 2-4 Shirane Shirakata Tokai-mura, \\ 319-1195, Japan \\ ${ }^{c}$ Elements Strategy Initiative for Structural Materials (ESISM), Kyoto University Yoshida, Honmachi, \\ Sakyo-ku, Kyoto 606-8501, Japan \\ ${ }^{d}$ Department of Quantum Science and Energy Engineering, Tohoku University 6-6-01-2 Aramaki-aza-Aoba \\ Aoba-ku Sendai, 980-8579, Japan
}

\begin{abstract}
Tungsten (W) is considered as a promising candidate for plasma-facing materials for future nuclear fusion devices, and selecting optimal alloying constituents is a critical issue to improve radiation resistance of the $\mathrm{W}$ alloys as well as to improve their mechanical properties. We conducted in the current study a series of first-principles calculations for investigating solvent-solute mixed dumbbells in $\mathrm{W}$ crystals. The results suggested that titanium (Ti), vanadium $(\mathrm{V})$, and chromium $(\mathrm{Cr})$ are favorable as solutes for $\mathrm{W}$ alloys from irradiationeffect perspectives because these elements are expected to promote vacancy-interstitial recombination without causing radiation-induced precipitation that reduces ductility of irradiated materials.
\end{abstract}

Key words: first-principles method, W alloys, mixed dumbbells, low-symmetrical configuration, migration path

\section{Introduction}

Tungsten (W) metals and alloys are considered as a promising candidate for plasmafacing materials for future nuclear fusion devices because of its high melting temperature, high resistance to sputtering, and high thermal conductivity. See related review articles $[1,2]$ and the references therein for detail. However, radiation-induced effects, such as swelling [3] and increase in deuterium retention [4] seem to threaten its actual applicability. Generally speaking, primary damage of neutron and ion irradiation to crystalline materials is the displacement of countless atoms from their original lattice positions; this causes many interstitial atoms, which become important players of the microstructural evolution. So far, a

\footnotetext{
*Corresponding author. Tel.: +81292825434

Email address: suzudo.tomoaki@jaea.go.jp (Tomoaki Suzudo)

Preprint submitted to Journal of Nuclear Materials
}

March 29, 2018 
lot of knowledge on interstitials not only in W crystals but also in other body-centered-cubic (BCC) transition metals have been accumulated. For instance, the most favored position of the interstitial atoms depends on element of interstitial: interstitials of light elements, such as hydrogen, helium, carbon, and oxygen, locate at octahedral site or tetrahedral site, on the other hands those of transition metals including self-interstitial atoms (SIAs) favor forming dumbbells, i.e., two atoms share a lattice site. Accordingly, heavy atoms in W crystals displaced by irradiation usually forms dumbbells. It had been conventionally assumed that such dumbbells would be configured in some symmetrical directions such as in $<111>,<110>$, or $<100>$ [5]. For example, $<111>$ SIA dumbbells in W crystals used to be recognized as the most energetically-favored configuration [6], and such SIAs would give one-dimensional (1D) motion: An atom in a dumbbell jumps to the first nearest neighboring $(1 \mathrm{NN})$ site, and it forms another dumbbell there; in the next step the atom initially located at the $1 \mathrm{NN}$ site jumps to the next leaving the atom that came to the site in the previous step. Currently, SIA dumbbells in a low-symmetrical direction between $<111>$ and $<110>$ are believed to be the most energetically-favored in W crystals [7]; they are called a $<11 \mathrm{~h}>$ dumbbell [7] or a bridge dumbbell [8]. Note that the 1D migration described above is practically observable even for the low-symmetrical SIAs [9] (see also Fig. 1).

In the case of irradiation of $\mathrm{W}$ alloys, there are two possible consequences for displaced W atoms (i.e., an SIA): If the SIA and a solute substitutional are attractive, they form a stable solvent-solute dumbbell after the SIA migrates and reaches the vicinity of the solute within the reaction radius; on the other hand, if these are repulsive to each other the displaced $\mathrm{W}$ atoms keep migrating without forming the mixed dumbbells. Rhenium (Re) and Osmium (Os) are produced from W through nuclear transmutation, and they would naturally be solute elements of W crystals under neutron irradiation. In such an alloy, such stable solvent-solute mixed dumbbells appear because the SIA and Re and Os substitutional atoms are attractive. Our previous study [7] analyzes the atomistic migration patterns of these two transmuted elements and indicates that the mixed dumbbells composed of Re (or Os) and $\mathrm{W}$ atoms are separated, and the Re (or Os) atom jumps to the $1 \mathrm{NN}$ site forming another mixed dumbbell. As discussed in the previous work [7, 11], if only this kind of events is allowed, possible migration paths are extremely limited. For example, a mixed dumbbell in $<111>$ seemingly jumps back and forth between two neighboring sites. However, this is not the case because rotation of these mixed dumbbells is commonly easy; these solute elements can be carried for long distance by iteration of jumping and rotation events, letting them be capable of fast three-dimensional (3D) migration [11]. These findings significantly changed the landscape of the solute migration in W, because such SIA-Re complexes had been thought as an immobile defect [12]. Other solute interstitials such as vanadium (V) and titanium ( $\mathrm{Ti}$ ) interstitials in $\mathrm{W}$ crystals are also found to form stable mixed-dumbbells with a W atom [8] and to migrate easily keeping the form of mixed dumbbells [13] as described above.

Re and Os experimentally proved to suppress radiation-induced defects [3, 14-19]. Through comprehensive theoretical discussions based on various pieces of experimental evidence [9], the 3D fast migration mentioned above seems to an origin of this phenomenon; this suggests that other solute elements may cause the similar effect. To optimize the property of W 


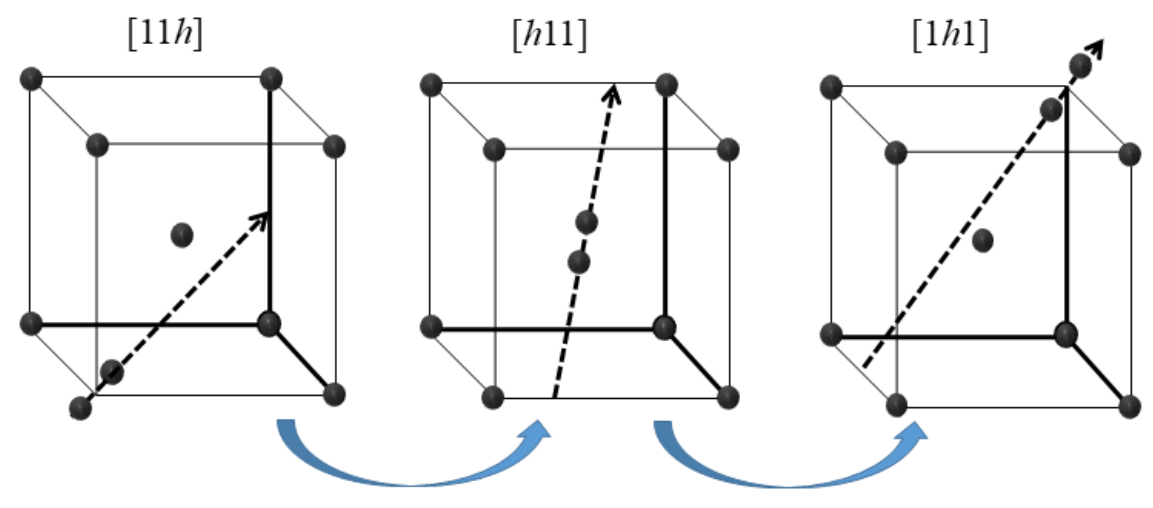

Figure 1: An example of 1D migration path of bridge dumbbell SIA: Despite of the fluctuation of the dumbbell direction, the migration paths becomes linear [9] as observed in experiments [10]. 
alloys as plasma-facing materials it is critical to explore the long-time kinetics of various solute elements under irradiation, but such a study is still halfway; kinetics of only Re and Os have been partly examined so far $[9,11,13,20]$. For this reason, parameters for various atomistic events related to many possible solute elements are indispensable.

In this paper, we examined stability and mobility of various solute interstitials in $\mathrm{W}$ crystals to search for candidates of solute elements of radiation-resistant $\mathrm{W}$ alloys. To be more specific, we intended to give comprehensive information necessary to the kinetic modeling of solute behavior under irradiation. In the current study, chromium $(\mathrm{Cr})$ and molybdenum (Mo) interstitials were found to form stable mixed-dumbbells in addition to Re, Os, Ti, and $\mathrm{V}$ interstitials, which are previously known as solutes forming stable mixed-dumbbells $[7,8]$. The energetically-favored direction of the stable dumbbells was a low-symmetrical one between $<111>$ and $<110>$, except $<110>$ W-Os mixed dumbbells. Further detailed first-principles study suggested that $\mathrm{Ti}, \mathrm{V}$, and $\mathrm{Cr}$ would be favorable alloying elements for radiation-resistant $\mathrm{W}$ alloys.

\section{Computational methodology}

The density functional theory (DFT) is applied to conduct the first-principles calculations in the framework of generalized gradient approximation with projector-augmented wave (PAW) pseudopotentials [21] using the Vienna ab initio simulation package (VASP) [22]. We, within this framework, adopt Perdew-Burke-Ernzerhof pseudopotentials [23] from the VASP library. For all DFT calculations, we use a cut-off energy of $350 \mathrm{eV}$ for the planewave basis with a first-order Methfessel-Paxton scheme employing a smearing parameter of $0.1 \mathrm{eV}$. Supercells applied are composed of 250 BCC lattice sites, having a dimension of $(5 \mathrm{a} \times 5 \mathrm{a} \times 5 \mathrm{a})$, respectively, where $a$ is the lattice constant obtained by the volume relaxation DFT calculation. For this supercell, the Monkhorst-Pack $3 \times 3 \times 3 \mathrm{k}$-point meshes [24] is used to sample the Brillouin zone. The periodic boundary condition is applied to all three directions for each case. In each DFT calculation the total energy is obtained after relaxation of atomic positions without volume relaxation. Gharaee and Erhart [8] gave a systematic investigation how the formation energy of solute interstitial in W crystals changes in different size of super cells. Their results indicate that the estimated values increase as the system size increases, and that the increase rate does not vary with types of interstitials: the equation to calculate binding energy of an SIA to a solute atom from total energy values is

$$
E_{b}^{S I A, \alpha}=E_{f}^{S u b-\alpha}+E_{f}^{S I A}-\min \left(E_{f}^{S I A, \alpha}\right),
$$

where $E_{f}^{S u b-\alpha}$ is the formation energy of $\alpha$-element substitutional atom; $\min \left(E_{f}^{S I A, \alpha}\right)$ is the formation energy of the most energetically-favored SIA-solute complex, and we exclusively assumed that the most energetically-favored form of this complex is a solvent-solute mixeddumbbell in the direction between $<111>$ and $<110>$ from our previous DFT study [7]. Note that the minimum formation energy of each mixed dumbbell is calculated not by nudged elastic band method explained below but by ordinary independent relaxation. Likewise, to calculate binding energy of a vacancy to a solute atom is given by:

$$
E_{b}^{\text {vacancy, } \alpha}=E_{f}^{S u b-\alpha}+E_{f}^{\text {vacancy }}-E_{f}^{\text {vacancy, } \alpha},
$$


where $E_{f}^{\text {vacancy }}$ and $E_{f}^{\text {vacancy- } \alpha}$ are vacancy and vacancy-solute complex formation energy values, respectively. Note that any binding energies obtained here do not depend upon the cohesive energy of solute element. As mentioned above the binding energy is evaluated from the difference between two formation energy values for different configuration of interstitials. So large part of the system size effect is canceled out by this subtraction, and the system size effect, i.e., the constant volume effect of the binding energy would not be significant.

The definition of a migration energy is the energy barrier between two stable atomic configurations. To evaluate this, the energy pathway between these two states is calculated using the nudged elastic band method, whose functionality is fully embedded in VASP. For all energy barrier calculations, we considered five intermediate points (or images) along the pathway, each of which was also calculated under the constant volume condition. Migration energy is also evaluated from the difference between two formation energy values, then we expect the constant volume effect is not significant either. As solute elements, we selected group 4, 5 and 6 transition metallic elements, Ti, V, Cr, zirconium (Zr), niobium (Nb), Mo, hafnium (Hf), tantalum ( Ta) and elements produced by nuclear transmutation in $\mathrm{W}$ under neutron irradiation, i.e., Re and Os. Note that we used the results in our previous paper [9] for the cases of Re and Os when available. These elements cover a broad range of alloying possibility in W. We used standard pseudopotentials in VASP library except for $\mathrm{Nb}$, for which a pseudopotential treating $p$ semi-core states as valence states was used. Because we confirmed magnetic calculations for $\mathrm{Cr}$ interstitials in $\mathrm{W}$ crystals made no meaningful difference from the paramagnetic ones, all the calculations were done in the paramagnetic state.

\section{Results}

\subsection{Stability of mixed dumbbells}

First of all, we discuss the stability of mixed dumbbells; the binding energy of each mixed dumbbell is summarized in Fig. 2 with vacancy-solute binding energy that will be discussed later in this paper. According to the figure, the mixed dumbbells are stable when the solute atom in the pair is $\mathrm{Ti}, \mathrm{V}, \mathrm{Cr}, \mathrm{Mo}, \mathrm{Re}$, or Os. In addition to previously-known stable mixed dumbbells, W-Re, W-Os, W-Ti, W-V dumbbells, we found that W-Cr and W-Mo pairs also form stable mixed-dumbbells, while Zr, Nb, Hf, and Ta do not form stable mixed-dumbbells, and are easy to separate into an SIA and a substitutional. We found that all the three Period-4 elements, i.e., Ti, V, and Cr form stable mixed dumbbell, while no clear tendency was found for Period-5 and -6 elements. In addition, we observed the binding energy tends to increase as the group number does.

In the following, we will exclude the discussion on the mixed dumbbells with the negative (repulsive) binding energy, because those dumbbells are hard to be created, and their lifetime would be extremely short even if they were created. The energy map of various solvent-solute dumbbells during the rotation from $<111>$ through $<110>$ is shown in Fig. 3. The lowsymmetrical direction is the most energetically-favored for all the mixed dumbbells with the exception of W-Os mixed dumbbells being the most stable at $<110>$ direction. If the direction of the most energetically-favored dumbbells is represented by $<11 h\rangle, h$ is between 


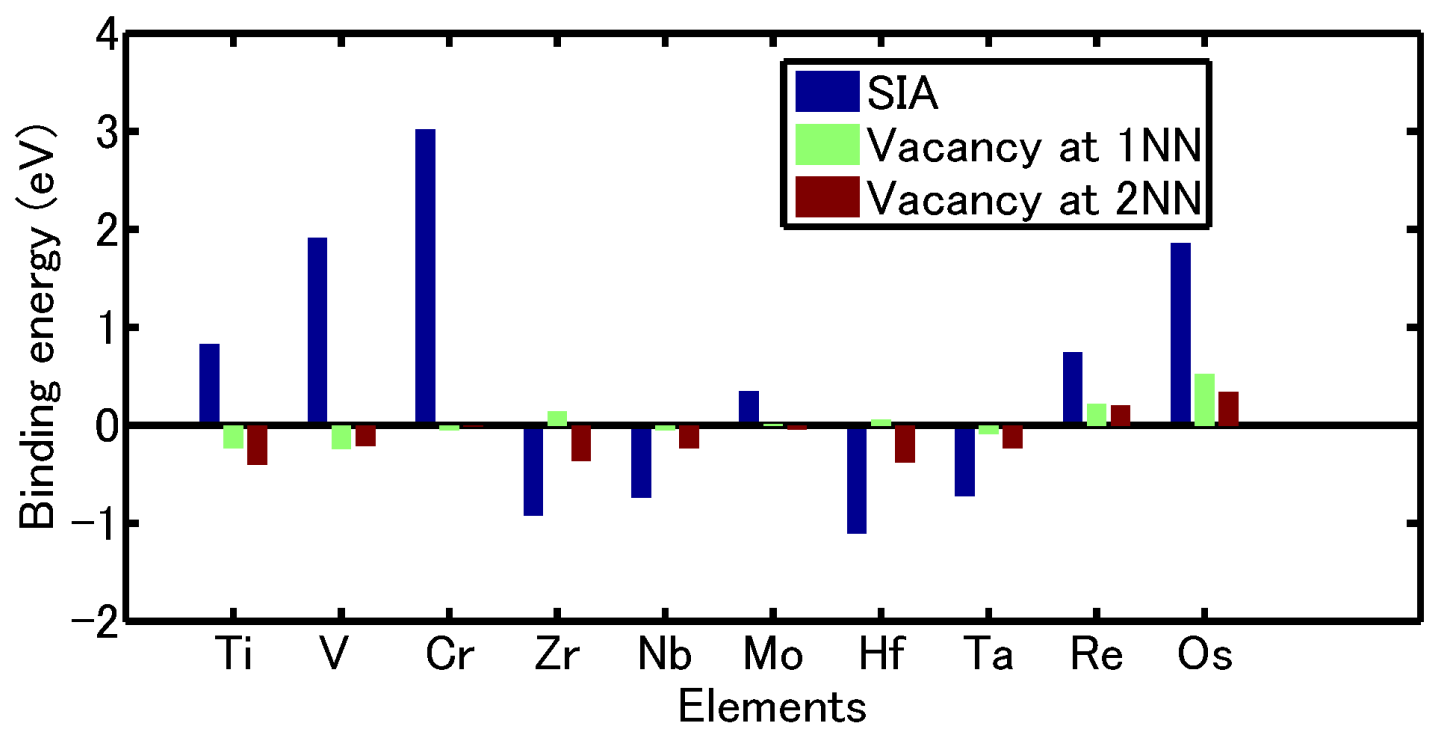

Figure 2: Binding energy between various solute atoms and an SIA, and between the solute atoms and a vacancy. All the values shown here are given by the current study except for the Os case, for which we used the results from the previous study [9].

0.3-0.7, the exact value being dependent on the solute element. The energy depression at $<11 h>$ from other symmetrical directions is also dependent on the solute element. Note that the influence of elastic interaction along the $\langle 111\rangle$ direction on the stability of the $<111>$ dumbbell is thoroughly discussed in a previous work using non-cubic supercells [7] and confirms that there is no influence on the equilibrium structure of the mixed dumbbells.

\subsection{Mobility of mixed dumbbells}

As mentioned above, we only analyze stable mixed-dumbbells, for which positive energy is necessary to separate them into an SIA and a solute substitutional atom. We can ignore this separation event for the solute migration analyses because solute substitutionals are not mobile by themselves; this section concentrates on the atomistic events in the form of mixed dumbbell whose outline is already mentioned in Introduction, i.e., it is composed of solute atom's jump to $1 \mathrm{NN}$ position and rotation of the dumbbell.

For the mixed dumbbells jumping to a $1 \mathrm{NN}$ site, three paths can be considered in the cases that the bridge dumbbell is the most energetically-favored, as illustrated in Fig. 4a, that is (1) $\rightarrow$ (2) $\rightarrow$ (1)', (3), and (4) $\rightarrow$ (5) $\rightarrow$ (4)'. In the case that the $<110>$ dumbbell is the most stable, practically only one path (5) is possible. The results given by DFT calculations for all the events, (1)-(5), are summarized in Table 1. The energy barriers for (1) $\rightarrow$ (2) $\rightarrow$ (1)', (3), and (4) $\rightarrow$ (5) $\rightarrow$ (4)' are $E_{1}+E_{2}, E_{3}$, and $E_{4}+E_{5}$, respectively. Notice that the easiest migration path for Ti and Re interstitials go through a $<110>$ direction before the jump to $1 \mathrm{NN}$ site, that is, the (4) $\rightarrow$ (5) $\rightarrow$ (4)' path. As shown in Fig. 3, the depression of the energy map is shallow for $\mathrm{Ti}$ and Re, and the bridge and $<110>$ directions are almost degenerated, 


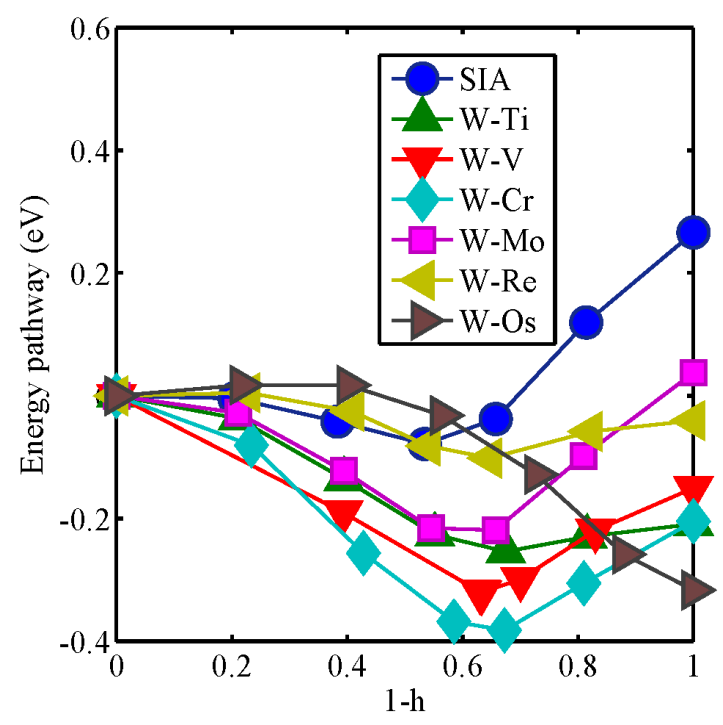

Figure 3: Energy map of the mixed dumbbells between $<111>$ and $<110>$ as a function of $h$ of the $<11 h>$ representation.

and (4) $\rightarrow$ (5) $\rightarrow$ (4)' path is almost equivalent to (3) within the accuracy of the DFT calculation. For the other elements, the direct jump path (3) are the energetically-easiest. Notice that all the migration energy values, $E_{3}$ s are similar, i.e., between $0.12 \mathrm{eV}$ and $0.16 \mathrm{eV}$ although each energy map between $<111>$ and $<110>$ is unique as shown in Fig. 3.

Next, we discuss mixed dumbbells in the bridge direction rotating over a $<110>$ direction and stabilizing as another bridge dumbbell. Fig. 4b is a 3D image of typical directions of dumbbells. Note that only one rotation path per bridge dumbbell over a $<110>$ direction is possible, such as $[11 h] \rightarrow[110] \rightarrow[11 \bar{h}]$. In fact, our NEB calculations confirmed that the easiest rotation path never bypath the $\langle 110\rangle$ direction. As shown in Fig. 5, this rotation let a mixed dumbbell migrate to a second nearest neighboring $(2 \mathrm{NN})$ site and is necessary to the long-distance migration. Note that mixed dumbbells, which has the lowest formation energy at the $<110>$ direction, are naturally possible to migrate to a $2 \mathrm{NN}$ site as this rotation event is not necessary. This rotation barrier energy is obtained by the excess value of the dumbbell's formation energy at the $\langle 110\rangle$ direction from that at the bridge direction, corresponding to the event (4) in Fig. 4a. The results are given as $E_{4}$ in Table 1; the values are scattered over the range of $0.04-0.26 \mathrm{eV}$ because of the unique energy pathway as shown in Fig. 3.

As you notice from Fig. 6, if the rotation over the $<111>$ in addition to the $<110>$ direction is allowed, solute atoms can migrate in the direction opposite to that in Fig. 5. As shown in Fig. 4b, three bridge dumbbells are in the vicinity of a $<111>$ dumbbell, and there are two equivalent rotation events from a bridge dumbbell over a $<111>$ direction. If these two events are allowed in addition to the rotation event over $<110>$ direction, solute atoms can migrate in any direction. Note that our DFT calculation confirm that the easiest rotation path of this type never bypath the $<111>$ direction. This event corresponds to (1) 
(a)

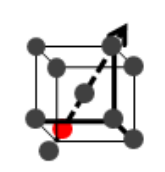

[111]

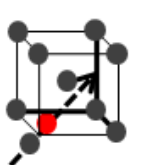

[11h]

(1)

(2)

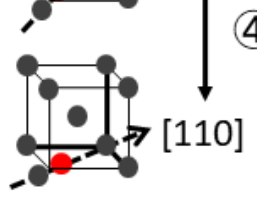

(4)

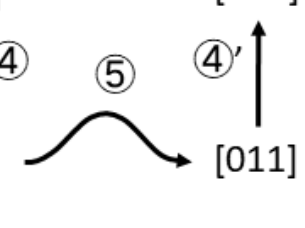

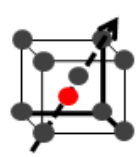
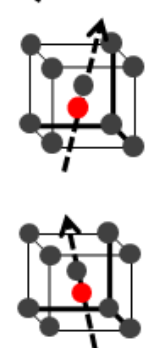

(b)

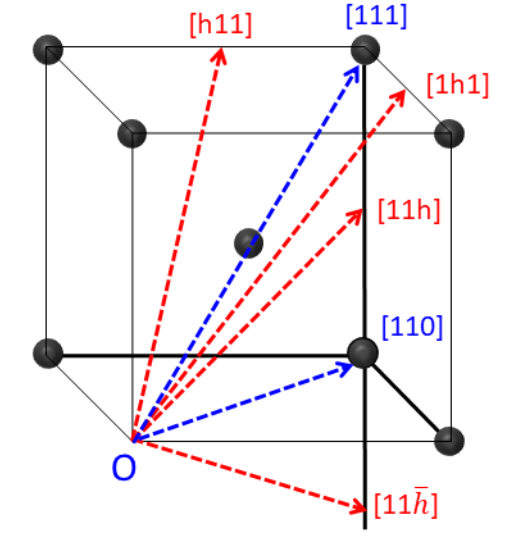

Figure 4: (a)The migration paths of mixed dumbbells: the red circles indicate solute atoms, (b) Schematic

diagram of dumbbell's direction in $\mathrm{W}$ crystals including low symmetrical ones denoted by red arrows

$[11 h]$

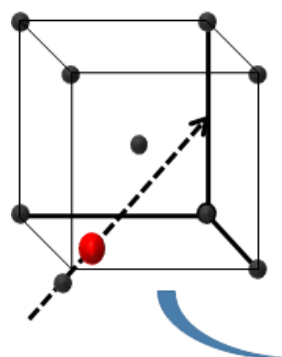

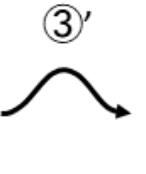

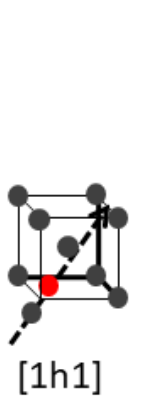

[h1]

\section{[1h1]}

direction enable this migration path. 


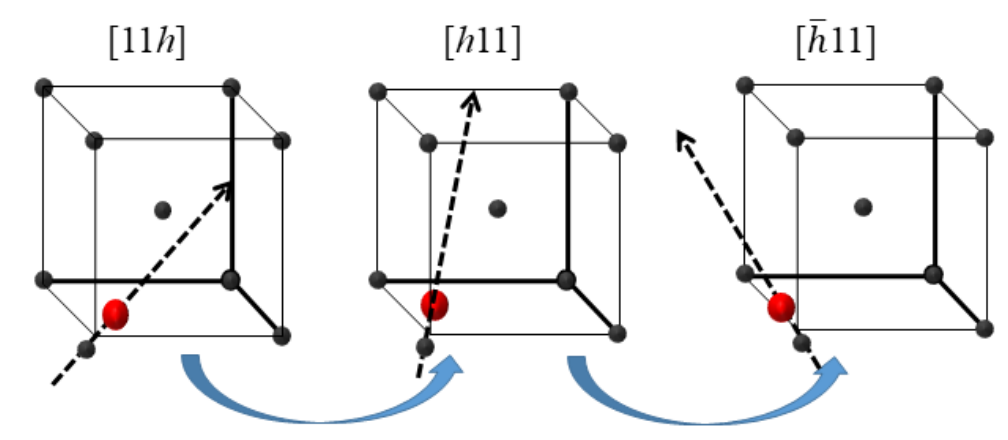

Figure 6: Migration path of a solute atom denoted by a red circle to the 2NN site: Rotation beyond [101] direction in addition to [111] direction remove the restriction of migration behaviors.

Table 1: Migration and rotation barrier energy $(\mathrm{eV})$ of stable solvent-solute mixed dumbbells: $E_{i}(i=1,5)$ is the barrier energy for event (1).

\begin{tabular}{llllll}
\hline & $E_{1}$ & $E_{2}$ & $E_{3}$ & $E_{4}$ & $E_{5}$ \\
\hline W-Ti & 0.25 & 0.02 & 0.15 & 0.04 & 0.10 \\
W-V & 0.32 & No barrier & 0.13 & 0.17 & No barrier \\
W-Cr & 0.39 & No barrier & 0.12 & 0.18 & No barrier \\
W-Mo & 0.23 & No barrier & 0.13 & 0.27 & Not converged \\
W-Re & 0.10 & 0.14 & 0.16 & 0.06 & 0.10 \\
\hline
\end{tabular}

in Fig. 4a. Notice that, in addition to the pure rotation path over the $<111>$ direction with the barrier energy being $E_{1}$, the path can be substituted by the consecutive back-and-forth jumps, (3) $\rightarrow$ (3)' with the barrier energy of each jump being $E_{3}$. Table 1 indicates that the easiest path only for W-Re dumbbells is the pure rotation because $E_{1}<E_{3}$ is satisfied, and for the remaining bridge dumbbells the alternative consecutive back-and-forth jumps are the easier. An important lesson given here is that the migration path of the solvent-solute dumbbell is dependent on the solute element.

\section{Discussion}

The results in the previous section indicated that all the stable solvent-solute mixed dumbbells shown in Table 1 can easily migrate with 3D motion. In our previous study [9], the mechanism how solute elements suppress radiation-induced defects is discussed, and a verylikely cause of this phenomenon is this 3D migration that increases the vacancy-interstitial recombination probability compared with $1 \mathrm{D}$ migration of SIAs in pure W. For example, we already know that Re and Os produced from W by nuclear transmutation reactions under neutron irradiation able to reduce surviving defects as described above. Demerit of these elements as alloying constituents is that they cause radiation-induced precipitation (RIP) that leads to hardening and embrittlement of the materials [14, 15, 18, 25]. Full picture of nucleation and growth of the RIP are still under discussion, but people generally believe that attractive binding energy between vacancy and a solute substitutional is essential as well as attractive binding energy between an SIA and the solute substitutional [20, 26, 27], 
because the vacancy and interstitial, both of which drag solute atoms, recombine to each other and cause the aggregation of solute atoms.

So far we have been discussing about vacancies recombining single isolated interstitials, but under realistic situation it is possible for vacancies to annihilate at different locations, such as at the boundary of precipitates, grain boundaries, dislocations, interstitial clusters. The recombinations at these locations are discussed in our previous study [9], and through various experimental data $[16-18,28]$ we had reached the conclusion that the suppression of radiation effects in experiments cannot be explained without the influence from the 3D motion of the mixed dumbbells, although we do not deny the possibility that other defects work as recombination sites and alleviate the radiation effects.

Fig. 2 shows binding energy $(\mathrm{eV})$ of solute substitutionals and vacancy at the first nearest neighbor $(1 \mathrm{NN})$ and the second nearest neighbor $(2 \mathrm{NN})$. These results well agree with the previous study $[29,30]$. Re and Os, which cause RIP in W crystals, have all positive (attractive) binding energies as expected, while Ta, which does not cause RIP [31], do not have any positive values. $\mathrm{Ti}, \mathrm{V}$, and $\mathrm{Cr}$ have repulsive interaction to vacancies, and accordingly, these are possible alloying elements which would be expected to reduce the irradiation effect without causing RIP. The discussion here does not necessarily suggest that $\mathrm{Ti}, \mathrm{V}$, and $\mathrm{Cr}$ are the best alloying elements of $\mathrm{W}$ for fusion application. $\mathrm{W}$ is so brittle at low temperatures that its ductile-to-brittle transition temperature (DBTT) is in the range of $250^{\circ} \mathrm{C} \sim 600^{\circ} \mathrm{C}\left[32\right.$ ] and that it reaches as high as $\sim 700^{\circ} \mathrm{C}$ after $\sim 1$ dpa irradiation [33]. Alloying of $\mathrm{W}$ often changes elastic properties [34, 35], and it also causes hardening $[36,37]$ as solute atoms may become obstacles for dislocation motion [38]; especially pure W is already highly brittle. We already know that Re improves the ductility of $\mathrm{W}$ materials at low temperatures [39], but its mechanism is still unclear although some limited theoretical work exists [40-42]. Besides, we ought to avoid solute elements that cause radioactive products under irradiation as much as possible. Consequently, the alloying elements in W crystal applied for plasma-facing materials must be selected from various perspectives.

As described above, 3D motion of the mixed dumbbells is the most probable reason of the suppression of the radiation effect in dilute alloys, while pure solute dumbbells may appear more concentrated alloys. These dumbbells are formed from the binding of a mobile solute-tungsten mixed-dumbbell and an immobile solute substitutional, so we expect the formation of Ti-Ti, Cr-Cr, Mo-Mo, Re-Re, and Os-Os dumbbells. Fig. 7 is the energy map of the pure solute dumbbells between $<111>$ and $<110>$ directions and indicates that the bridge direction is energetically most favored for $\mathrm{Cr}-\mathrm{Cr}$ and Mo-Mo dumbbells, while the $<110>$ direction is energetically most favored for the remaining pure solute dumbbells. Fig. 8 shows the binding energy of each pure solute dumbbell at the most energetically favored direction. If a binding energy of pure solute dumbbells is larger than vacancy migration energy, the recombination would be regulated by the $3 \mathrm{D}$ vacancy migration rather than $3 \mathrm{D}$ mixed-dumbbell migration. As shown in Fig. 8, the mixed-dumbbell migration mechanism is dominant for $\mathrm{Ti}, \mathrm{Mo}$, and Re cases, while the vacancy migration mechanism is dominant for $\mathrm{V}, \mathrm{Cr}$, and Os cases. Whichever the recombination mechanism is dominant, 3D migration reduce the surviving defects in comparison to the $1 \mathrm{D}$ motion of SIAs.

According to Fig. 3, the energy depression at the formation energy of the stable mixed- 


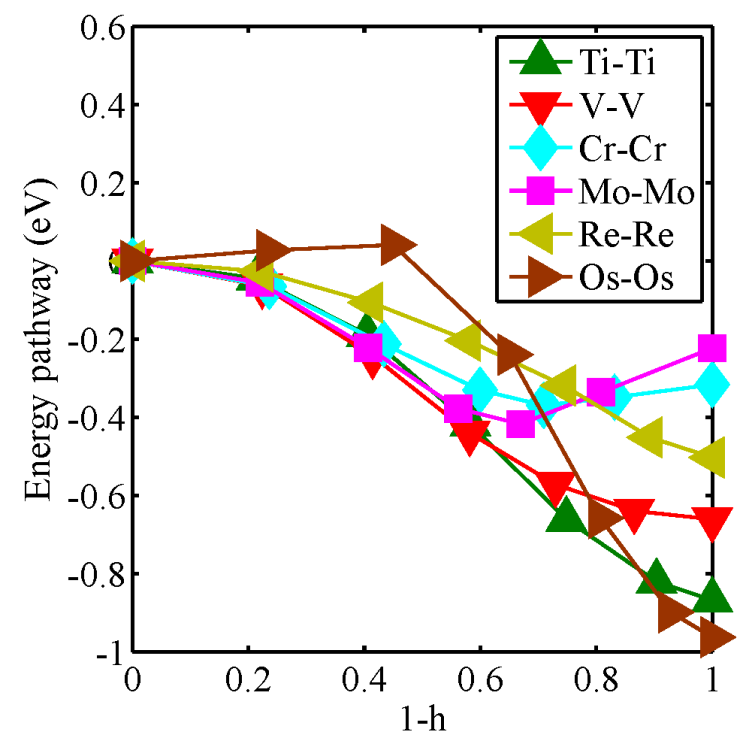

Figure 7: Energy map of the pure solute dumbbells between $<111>$ and $<110>$ as a function of $h$ of the $<11 h>$ representation.

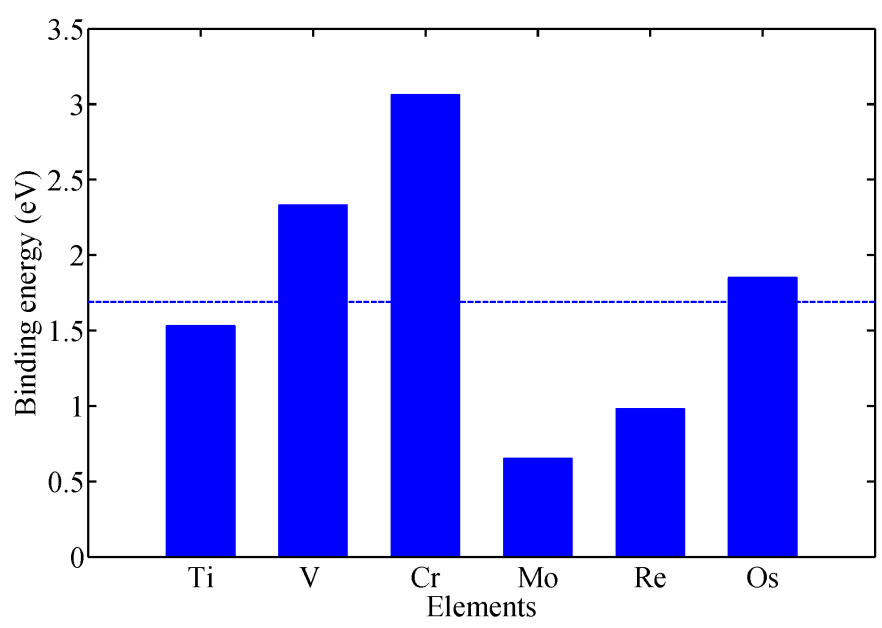

Figure 8: Binding energy of pure solute dumbbells: the level line shows the vacancy migration energy. 


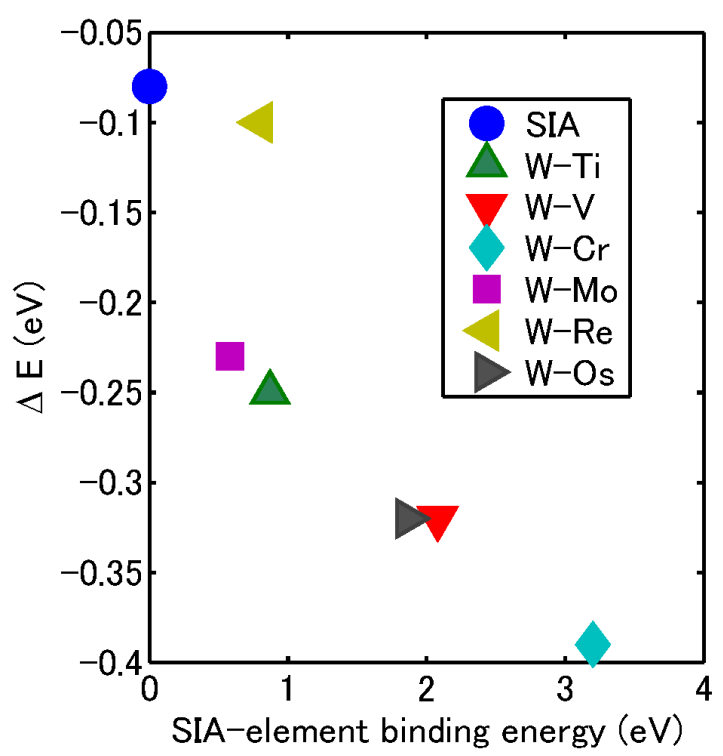

Figure 9: Excess energy of the most energetically-favored direction from the energy of $<111>$ direction as a function of the binding energy of the most energetically-favored mixed dumbbells; Note that binding energy of SIA is zero.

dumbbells at the low-symmetrical direction becomes meaningfully larger than that of $<111>$ dumbbells (i.e. crowdions) compared with the SIA dumbbell whose binding energy is nil; this implies that the binding energy should contribute to the stability of the non-crowdion type dumbbells. To confirm this hypothesis the excess energy of the non-crowdion dumbbells from that of the crowdion is represented as a function of the binding energy between an SIA and each solute (Fig. 9): The diagram indicates that the relative stability of the noncrowdion dumbbells is dependent on the binding energy. This suggests that the departure from the $<111>$ direction is caused not by the repelling interaction along the compressive $<111>$ direction but by the attractive interaction between the dumbbell and atoms in tensile $<111>$ directions. The above notion is still speculative and must be confirmed by more detailed analyses in the future.

\section{Conclusions}

In this paper, we examined the stability of solvent-solute mixed dumbbells in $\mathrm{W}$ crystals by using first-principles DFT calculations, and $\mathrm{Cr}$ and Mo interstitials were found to form stable solvent-solute mixed-dumbbells in addition to Re, Os, Ti, and V interstitials, which are already known as stable mixed-dumbbells. The most energetically-favored direction of all the stable dumbbells except $<110>\mathrm{W}$-Os mixed dumbbells were low-symmetrical one between $<111>$ and $<110>$. The discussion in the present study suggests that $\mathrm{Ti}, \mathrm{V}$, and $\mathrm{Cr}$ would be favorable alloying elements from the irradiation-effect perspectives because these are expected to promote vacancy-interstitial recombination without causing precipitation. The DFT data given here is essential information for kinetic modeling of radiation-induced 
microstructural evolution, and such modeling is necessary for computationally pursuing optimal $\mathrm{W}$ alloys for plasma-facing materials.

\section{Acknowledgements}

A part of this work is supported by JSPS KAKENHI Grant Number 15K06672. A part of this work is carried out using the HELIOS supercomputer system at Computational Simulation Centre of International Fusion Energy Research Centre (IFERC-CSC), Aomori, Japan under the Broader Approach collaboration between Euratom and Japan, implemented by Fusion for Energy and JAEA.

\section{References}

[1] M. Rieth, S. Dudarev, S. Gonzalez de Vicente, J. Aktaa, T. Ahlgren, S. Antusch, D. Armstrong, M. Balden, N. Baluc, M.-F. Barthe, et al., Recent progress in research on tungsten materials for nuclear fusion applications in europe, Journal of Nuclear Materials (2012).

[2] J. Marian, C. S. Becquart, C. Domain, S. L. Dudarev, M. R. Gilbert, R. J. Kurtz, D. R. Mason, K. Nordlund, A. E. Sand, L. L. Snead, et al., Recent advances in modeling and simulation of the exposure and response of tungsten to fusion energy conditions, Nucl. Fusion 57 (2017) 092008.

[3] J. Matolich, H. Nahm, J. Moteff, Swelling in neutron irradiated tungsten and tungsten-25 percent rhenium, Scripta Metallurgica 8 (1974) 837-841.

[4] Y. Hatano, M. Shimada, V. K. Alimov, J. Shi, M. Hara, T. Nozaki, Y. Oya, M. Kobayashi, K. Okuno, T. Oda, et al., Trapping of hydrogen isotopes in radiation defects formed in tungsten by neutron and ion irradiations, Journal of Nuclear Materials 438 (2013) S114-S119.

[5] R. Johnson, Interstitials and vacancies in $\alpha$ iron, Physical Review 134 (1964) A1329.

[6] D. Nguyen-Manh, A. P. Horsfield, S. L. Dudarev, Self-interstitial atom defects in bcc transition metals: Group-specific trends, Physical Review B 73 (2006) 020101.

[7] T. Suzudo, M. Yamaguchi, A. Hasegawa, Stability and mobility of rhenium and osmium in tungsten: first principles study, Modelling and Simulation in Materials Science and Engineering 22 (2014) 075006.

[8] L. Gharaee, P. Erhart, A first-principles investigation of interstitial defects in dilute tungsten alloys, Journal of Nuclear Materials 467 (2015) 448-456.

[9] T. Suzudo, A. Hasegawa, Suppression of radiation-induced point defects by rhenium and osmium interstitials in tungsten, Scientific reports 6 (2016) 36738.

[10] T. Amino, K. Arakawa, H. Mori, Detection of one-dimensional migration of single self-interstitial atoms in tungsten using high-voltage electron microscopy, Scientific reports 6 (2016).

[11] T. Suzudo, M. Yamaguchi, A. Hasegawa, Migration of rhenium and osmium interstitials in tungsten, Journal of Nuclear Materials 467 (2015) 418-423.

[12] K. Wilson, M. Baskes, D. Seidman, An in situ field-ion microscope study of the recovery behavior of ion-irradiated tungsten and tungsten alloys, Acta Metallurgica 28 (1980) 89-102.

[13] L. Gharaee, J. Marian, P. Erhart, The role of interstitial binding in radiation induced segregation in w-re alloys, Journal of Applied Physics 120 (2016) 025901.

[14] R. Herschitz, D. N. Seidman, Radiation-induced precipitation in fast-neutron irradiated tungstenrhenium alloys: An atom-probe field-ion microscope study, Nuclear Instruments and Methods in Physics Research Section B: Beam Interactions with Materials and Atoms 7 (1985) 137-142.

[15] T. Tanno, A. Hasegawa, M. Fujiwara, J.-C. He, S. Nogami, M. Satou, T. Shishido, K. Abe, Precipitation of solid transmutation elements in irradiated tungsten alloys, Materials Transactions 49 (2008) 22592264.

[16] T. Tanno, M. Fukuda, S. Nogami, A. Hasegawa, Microstructure development in neutron irradiated tungsten alloys, Materials transactions 52 (2011) 1447-1451. 
[17] M. Fukuda, T. Tanno, S. Nogami, A. Hasegawa, Effects of re content and fabrication process on microstructural changes and hardening in neutron irradiated tungsten, Materials Transactions 53 (2012) 2145-2150.

[18] M. Fukuda, K. Yabuuchi, S. Nogami, A. Hasegawa, T. Tanaka, Microstructural development of tungsten and tungsten-rhenium alloys due to neutron irradiation in hfir, Journal of Nuclear Materials 455 (2014) 460-463.

[19] Y. Hatano, K. Ami, V. K. Alimov, S. Kondo, T. Hinoki, T. Toyama, M. Fukuda, A. Hasegawa, K. Sugiyama, Y. Oya, et al., Deuterium retention in w and w-re alloy irradiated with high energy fe and w ions: Effects of irradiation temperature, Nuclear Materials and Energy 9 (2016) 93-97.

[20] C.-H. Huang, L. Gharaee, Y. Zhao, P. Erhart, J. Marian, Mechanism of nucleation and incipient growth of re clusters in irradiated w-re alloys from kinetic monte carlo simulations, Physical Review B 96 (2017) 094108.

[21] G. Kresse, D. Joubert, From ultrasoft pseudopotentials to the projector augmented-wave method, Phys. Rev. B 59 (1999) 1758-1775.

[22] G. Kresse, J. Furthmüller, Efficient iterative schemes for ab initio total-energy calculations using a plane-wave basis set, Phys. Rev. B 54 (1996) 11169-11186.

[23] J. P. Perdew, K. Burke, M. Ernzerhof, Generalized gradient approximation made simple, Physical review letters 77 (1996) 3865.

[24] H. J. Monkhorst, J. D. Pack, Special points for brillouin-zone integrations, Physical Review B 13 (1976) 5188-5192.

[25] V. Sikka, J. Moteff, Identification of $\alpha$-mn crystal structure in neutron irradiated w-re alloy, Metallurgical and Materials Transactions B 5 (1974) 1514-1517.

[26] G. Martin, Contribution of dissipative processes to radiation-induced solid-solution instability, Physical Review B 21 (1980) 2122.

[27] R. Cauvin, G. Martin, Solid solutions under irradiation. i. a model for radiation-induced metastability, Physical Review B 23 (1981) 3322.

[28] A. Xu, C. Beck, D. E. Armstrong, K. Rajan, G. D. Smith, P. A. Bagot, S. G. Roberts, Ion-irradiationinduced clustering in $\mathrm{w}-\mathrm{re}$ and $\mathrm{w}-\mathrm{re}-\mathrm{os}$ alloys: A comparative study using atom probe tomography and nanoindentation measurements, Acta Materialia 87 (2015) 121-127.

[29] C. S. Becquart, C. Domain, U. Sarkar, A. DeBacker, M. Hou, Microstructural evolution of irradiated tungsten:i i i ab initioi/ic parameterisation of an okmc model, Journal of Nuclear Materials 403 (2010) $75-88$.

[30] X.-S. Kong, X. Wu, Y.-W. You, C. Liu, Q. Fang, J.-L. Chen, G.-N. Luo, Z. Wang, First-principles calculations of transition metal-solute interactions with point defects in tungsten, Acta Materialia 66 (2014) 172-183.

[31] A. Xu, D. E. Armstrong, C. Beck, M. P. Moody, G. D. Smith, P. A. Bagot, S. G. Roberts, Ion-irradiation induced clustering in w-re-ta, w-re and w-ta alloys: An atom probe tomography and nanoindentation study, Acta Materialia 124 (2017) 71-78.

[32] J. Davis, V. Barabash, A. Makhankov, L. Plöchl, K. Slattery, Assessment of tungsten for use in the iter plasma facing components, Journal of nuclear materials 258 (1998) 308-312.

[33] D. Stork, S. Zinkle, Introduction to the special issue on technical status of materials for a fusion reactor, Nucl. Fusion 57 (2017) 092001.

[34] R. Ayres, G. Shannette, D. Stein, Elastic constants of tungsten-rhenium alloys from 77 to $298 \mathrm{k}$, Journal of Applied Physics 46 (1975) 1526-1530.

[35] Y. Ma, Q.-F. Han, Z.-Y. Zhou, Y.-L. Liu, First-principles investigation on mechanical behaviors of w-cr/ti binary alloys, Journal of Nuclear Materials 468 (2016) 105-112.

[36] M. Aguirre, A. Martín, J. Pastor, J. Llorca, M. Monge, R. Pareja, Mechanical behavior of w-y2o3 and w-ti alloys from 25 c to 1000 c, Metallurgical and Materials Transactions A 40 (2009) 2283-2290.

[37] K. Arshad, M.-Y. Zhao, Y. Yuan, Y. Zhang, Z.-H. Zhao, B. Wang, Z.-J. Zhou, G.-H. Lu, Effects of vanadium concentration on the densification, microstructures and mechanical properties of tungsten vanadium alloys, Journal of Nuclear Materials 455 (2014) 96-100. 
[38] T. Tsuru, T. Suzudo, First-principles calculations of interaction between solutes and dislocations in tungsten, to be published in Nuclear Materials and Energy (2018).

[39] P. L. Raffo, Yielding and fracture in tungsten and tungsten-rhenium alloys, Journal of the Less Common Metals 17 (1969) 133-149.

[40] L. Romaner, C. Ambrosch-Draxl, R. Pippan, Effect of rhenium on the dislocation core structure in tungsten, Physical Review Letters 104 (2010) 195503.

[41] H. Li, S. Wurster, C. Motz, L. Romaner, C. Ambrosch-Draxl, R. Pippan, Dislocation-core symmetry and slip planes in tungsten alloys: $\mathrm{Ab}$ initio calculations and microcantilever bending experiments, Acta Materialia 60 (2012) 748-758.

[42] G. D. Samolyuk, Y. Osetsky, R. Stoller, The influence of transition metal solutes on the dislocation core structure and values of the peierls stress and barrier in tungsten, Journal of Physics: Condensed Matter 25 (2012) 025403. 